American Journal of Applied Sciences 6 (2): 276-284, 2009

ISSN 1546-9239

(C) 2009 Science Publications

\title{
Numerical Study of Airflow around Vehicle A-pillar Region and Windnoise Generation Prediction
}

\author{
${ }^{1}$ M.H. Shojaefard, ${ }^{2}$ K. Goudarzi and ${ }^{2}$ H. Fotouhi \\ ${ }^{1}$ Automotive Engineering Department, \\ ${ }^{2}$ Mechanical Engineering Department, \\ Iran University of Science and Technology, Tehran, Iran
}

\begin{abstract}
Flow separation and reattachment around the vehicle A-pillar region dictates strong pressure fluctuations on the side window surfaces and can also lead to generate aerodynamic noise. The objective of this work is to investigate qualitative flow visualization of airflow behaviour around vehicle A-pillar and its potential to generate windnoise in this region. By means of Computational Fluid Dynamic (CFD) under laboratory operating conditions, a series of three-dimensional NavierStokes simulations for the vortical flow around two simplified basic car models with different A-pillar/windshield geometry were carried out at different cruising speed. Both models were made with $60^{\circ}$ flat inclination angles but with deferent A-pillar/windshield curvature, a small semiellipsoidal shape, a slanted sharp-edged shape. Investigations were carried out at velocities 60,100 and 140 for 0 and 15 degrees yaw angles. Results of mean pressure coefficient obtaining using CFD modeling were also compared against available experimental data. Furthermore, using Boundary Layer Noise Source Model, an approximate measure of the local contribution to total acoustic power per unit surface area was carried out in a given turbulence field. The studies provided reasonable agreement against available experimental data. The studies show that the surface mean pressure coefficients magnitudes are independent of Reynolds numbers and dependent largely to A-pillar and windshield effective corner radii. In addition, surface acoustic power level analyses show that A-pillar and windshield local corner radii effects significantly to potential of noise generation around A-pillar region.
\end{abstract}

Keywords: A-pillar, A-pillar curvature, Yaw angle, Mean pressure coefficient, Aerodynamic noise

\section{INTRODUCTION}

As engine, tire, and other noise reduced and as driving speeds increased, aerodynamic noise sources on ground vehicles were becoming relatively more important. They often dominate at cruise speeds over $100 \mathrm{~km} \mathrm{~h}^{-1}$. Reduction of aerodynamic noise has a significant effect to occupants comfort on a long way trip. Consequently, the interior noise in a vehicle is important to its sales, particularly for luxury cars.

It is well known that the pressure fluctuations on the front side window surface of a road vehicle are a major sound source for both the external and interior wind noises ${ }^{[1]}$.

The Previous research studies revealed that the unsteady pressure on the front side window surface is due to the existence of three-dimensional vortex separated from the A-pillar region ${ }^{[1,2]}$. These researches also suggested that the size and magnitude of the
A-pillar flow separation mainly depend on the local Apillar and windshield geometry and vehicle yaw angles $[3,4,5]$. Consequently, in order to reduce wind noise, understanding the mechanics of airflow behaviour around the A-pillar region is very important.

In this study, a series of three-dimensional NavireStockes simulations were carried out for the vortical flow about basic car models with different Apillar/windshield geometry and at different cruising speed for different yaw angles; the models geometries were similar to $\operatorname{Alam}^{[6]}$. The airflow behaviour behind slanted-sharp edged model and semi-small ellipsoidal model were simulated using CFD and qualitatively analyzed. Pathlines analysis, contours of mean pressure coefficient and contours of turbulent kinetic energy helped to find out the characteristics of the airflow around the vehicle A-pillar. It is noted that the airflow simulation around the A-pillar region was investigated in this research.

Corresponding Author: M.H. Shojaefard, Department of Mechanical Engineering, Iran University of Science and Technology Narmak, 16844, Tehran, Iran Tel: ++98 21 73913963, Fax: ++98 2177491225 
In order to investigate windnoise around vehicle A-pillar, the turbulence quantities, which were acquired by CFD analysis, were used to estimate local sound source strength by using Boundary Layer Noise Source Model which is an acoustic source strength broadband noise model.

\section{VEHICLE GEOMETRY MODELS}

Geometry configuration used in these simulations were obtained from $\mathrm{Alam}^{[6]}$. In his wind tunnel tests, $40 \%$ scale idealized model vehicles with different Apillar and windshield radii were made. All models were simple without the complication of engine compartment, fore-body, side mirrors, wheels and wheel arches. In this study, two of them were selected which they are made with $60^{\circ}$ flat windshield inclination angles but with different A-pillar and windshield curvature; a slanted sharp-edged model, a small semi-ellipsoidal model. The full length of the model was $1.963 \mathrm{~m}$, the width was $0.748 \mathrm{~m}$ and the height was $0.588 \mathrm{~m}$ (Fig. 1).
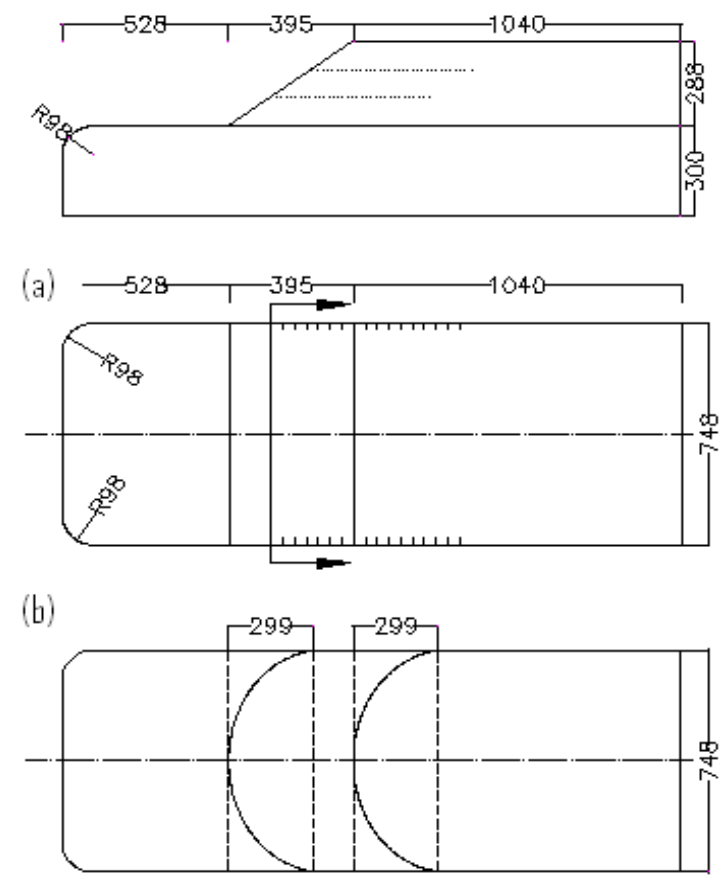

Fig. 1: The external dimension of simplified road vehicle (a) Top view (slanted sharp-edged model) (b) Top view (small ellipsoidal model)

\section{GOVERNING EQUATION}

Turbulent flow over the vehile A-pillar is threedimensional, steady and incompressible; the continuity and momentum equations (Navier-Stokes equations) with a turbulence model were used to solve the flow.

$$
\begin{aligned}
& \frac{\partial u}{\partial x}+\frac{\partial v}{\partial y}+\frac{\partial w}{\partial z}=0 \\
& u \frac{\partial u}{\partial x}+v \frac{\partial u}{\partial y}+w \frac{\partial u}{\partial z}=-\frac{1}{\rho} \frac{\partial p}{\partial x}+\frac{1}{\rho}\left(\frac{\partial \tau_{x y}}{\partial y}+\frac{\partial \tau_{x z}}{\partial z}\right)+B_{x} \\
& u \frac{\partial v}{\partial x}+v \frac{\partial v}{\partial y}+w \frac{\partial v}{\partial z}=-\frac{1}{\rho} \frac{\partial p}{\partial y}+\frac{1}{\rho}\left(\frac{\partial \tau_{x y}}{\partial x}+\frac{\partial \tau_{y z}}{\partial z}\right)+B_{Y} \\
& u \frac{\partial w}{\partial x}+v \frac{\partial w}{\partial y}+w \frac{\partial w}{\partial z}=-\frac{1}{\rho} \frac{\partial p}{\partial z}+\frac{1}{\rho}\left(\frac{\partial \tau_{x z}}{\partial x}+\frac{\partial \tau_{y z}}{\partial y}\right)+B_{z}
\end{aligned}
$$

Where $\mathrm{u}$ is $\mathrm{x}$-component of velocity vector, $\mathrm{v}$ is $\mathrm{y}$ component of velocity and $\mathrm{w}$ is $\mathrm{z}$-component of velocity vector. $\rho$ is density of air, $P$ is static pressure, $\tau$ is shear stress and $B_{x}, B_{y}, B_{z}$ are body forces ${ }^{[7]}$.

\section{TURBULENCE MODEL}

In this research, the turbulence model used for simulations was realizable $(\mathrm{K}-\varepsilon)$ model. The term realizable means that the model satisfies certain mathematical constraints on the normal stresses, consistent with the physics of turbulent flows. It provides superior performance for flows involving rotation, boundary layers under strong adverse pressure gradients, separation, and recirculation ${ }^{[8]}$, so realizable $(\mathrm{K}-\varepsilon)$ model is a proper model for visualization of airflow around vehicle A-pillar region. The modeled transport equations for $\mathrm{k}$ and $\epsilon$ in the realizable $(\mathrm{K}-\varepsilon)$ model are

$$
\begin{aligned}
& \frac{\partial}{\partial t}(\rho k)+\frac{\partial}{\partial x_{i}}\left(\rho k u_{j}\right)=\frac{\partial}{\partial x_{i}}\left[\left(\mu+\frac{\mu_{t}}{\sigma_{k}}\right) \frac{\partial k}{\partial x_{j}}\right] \\
& +G_{k}+G_{b}-\rho \varepsilon-Y_{m}+S_{k} \\
& \frac{\partial}{\partial t}(\rho \varepsilon)+\frac{\partial}{\partial x_{j}}\left(\rho \varepsilon_{u_{j}}\right)=\frac{\partial}{\partial x_{j}}\left[\left(\mu+\frac{\mu_{t}}{\sigma_{\varepsilon}}\right) \frac{\partial \varepsilon}{\partial x_{j}}\right] \\
& +\rho C_{1} S \varepsilon-\rho C_{2} \frac{\varepsilon^{2}}{k+\sqrt{v \varepsilon}}+C_{1 \varepsilon} \frac{\varepsilon}{k} C_{3 \varepsilon} G_{b}+S_{\varepsilon}
\end{aligned}
$$


Where,

$$
C_{1}=\max \left[0.43, \frac{\eta}{\eta+5}\right], \eta=S \frac{k}{\varepsilon}
$$

In these equations, $G_{k}$ represents the generation of turbulence kinetic energy due to the mean velocity gradients. $G_{b}$ is the generation of turbulence kinetic energy due to buoyancy. $\mathrm{Y}_{\mathrm{M}}$ represents the contribution of the fluctuating dilatation in compressible turbulence to the overall dissipation rate. $\mathrm{C}_{2}$ and $\mathrm{C}_{1 \varepsilon}$ are constants.

$\sigma_{\mathrm{k}}$ and $\sigma_{\varepsilon}$ are the turbulent Prandtl numbers for $\mathrm{k}$ and $\varepsilon$, respectively. $\sigma_{\mathrm{k}}$ and $\sigma_{\varepsilon}$ are user-defined source terms. The models constants $\mathrm{C}_{2}, \sigma_{\mathrm{k}}$ and $\sigma_{\varepsilon}$ have been established to ensure that the model performs well for certain canonical flows the model constants were

$$
\mathrm{C}_{1 \varepsilon}=1.44, \mathrm{C}_{2}=1.9, \sigma_{\mathrm{k}}=1.0, \sigma_{\varepsilon}=1.2
$$

The eddy viscosity was computed from

$$
\mu_{t}=\rho_{c_{\mu}} \frac{k^{2}}{\varepsilon}
$$

The difference between the realizable $(\mathrm{k}-\varepsilon)$ model and the standard and RNG $(\mathrm{k}-\varepsilon)$ models is that $\mathrm{C}_{\mu}$ is no longer constant. The $\mathrm{C}_{\mu}$ can be concluded form reference ${ }^{[9]}$.

\section{BROADBAND NOISE SOURCE MODEL}

In many practical applications involving turbulent flows, the sound energy is continuously distributed over a broad range of frequencies. In those situations involving broadband noise, Reynolds Averaged NavierStokes (RANS) equations can be utilizes to find the turbulence quantities. Consequently, these quantities can be used in conjunction with semi-empirical correlations and Lighthill's acoustic analogy to come up with some measures of the source of broadband noise. In this paper, the boundary layer noise source model was used in order to predict acoustic power level in side window surface.

Far field sound generated by turbulent boundary layer flow over a solid body at low Mach numbers is often of practical interest. The Curle's integral based on acoustic analogy can be used to approximate the local contribution from the body surface to the total acoustic power ${ }^{[10,11]}$.

The curls integral

$$
p^{\prime}(\vec{x}, t)=\frac{1}{4 \pi a_{0}} \int_{S} \frac{\left(\mathrm{x}_{\mathrm{i}}-y_{i}\right) n_{i}}{r^{2}} \frac{\partial p}{\partial t}(\vec{y}, \tau) d S(\vec{y})
$$

Where $\tau$ denotes the emission time and $\mathrm{S}$ is the integral surface

$$
\tau=t-r / a_{0}
$$

Using this, the sound intensity in the far field can then be approximated by

$$
\vec{p}^{\prime} \cong \frac{1}{16 \pi^{2} a_{0}^{2}} \int_{S} \frac{\cos ^{2} \theta}{r^{2}} \overline{\left[\frac{\partial p}{\partial t}(\vec{y}, \tau)\right]^{2} A_{C}(\vec{y})} d S(\vec{y})
$$

Where the $\mathrm{A}_{\mathrm{c}}$ is the correlation area, $\mathrm{r} \equiv|\overrightarrow{\mathrm{x}}-\overrightarrow{\mathrm{y}}|$ and the $\cos \theta$ is the angle between $|\vec{x}-\vec{y}|$ and the wall normal direction $\vec{n}$.

The total acoustic power emitted from the entire body surface can be computed from

$$
\begin{aligned}
& P_{A}=\frac{1}{\rho_{0} a_{0}} \int_{0}^{2 \pi \pi} \int_{0} \overline{p^{\prime 2}} r^{2} \sin \theta d \theta d \psi \\
& p_{A}=\int_{S} I(\vec{y}) d S(\vec{y})
\end{aligned}
$$

Where

$$
I(\vec{y}) \equiv \frac{A_{c}(\vec{y})}{12 \rho_{0} \pi_{a_{0}}^{3}} \overline{\left[\frac{\partial p}{\partial t}\right]^{2}}
$$

This can be interpreted as the local contribution per unit surface area of the body surface to the total acoustic power.

The mean square time derivative of the surface pressure and the correlation area are further approximated in terms of turbulent quantities like turbulent kinetic energy, dissipation rate and wall shear.

\section{BOUNDARY CONDITION}

The simulations were carried out in the virtual wind tunnel constructed in HFIUS software with full length of the tunnel was $10 \mathrm{~m}$, the width was $5 \mathrm{~m}$ and the height was $4 \mathrm{~m}$.The non-slip wall boundary condition is imposed on the car body. The velocity inlet is set at the inflow boundary and the pressure is set at zero at the outer boundary. In all of the simulations, the realizable $(\mathrm{K}-\varepsilon)$ turbulence model was used and calculation of the kinetic energy and dissipation rate $(\mathrm{K}-\varepsilon)$ were based on the turbulence intensity and 
turbulence length scale that were set at 1.8 and $1 \%$ of the model height respectively. These values varied accordingly with the inlet velocity.

\section{CONDITIONS OF SIMULATIONS}

In this research, a series of three-dimensional Navier-Stockes simulations were carried out for the flow around a simplified car model by the nonecommercial CFD code called HFIUS. The variables were the A-pillar and windshield geometry, Reynolds number (varied by cruising speed) and yaw angles. The cruising speed were set at 60,100 and $140 \mathrm{~km} \mathrm{~h}^{-1}$, which corresponded to the Reynolds number range of $2.17 * 10^{6}-5.03 * 10^{6}$ with the vehicle length as the length scale. Furthermore, simulations were carried out on both models at yaw angle 0 and 15 degrees.

\section{MESH GENERATION}

An advance meshing system used in this simulations that a blend of hexahedron grids was used to mesh and refine complex 3-D computational domain. In the first step, 650/000 grid cells was generated. The final mesh after refinement and adaption was slightly more than 1.4 million grid cells with 750,000 grid cells generated around A-pillar region (Fig. 2).

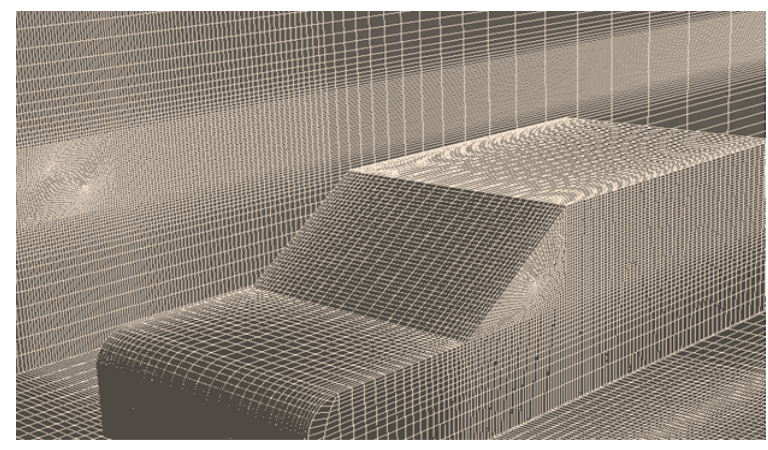

Fig. 2: The basic vehicle model and its grids

\section{NUMERICAL SCHEME AND STRATEGY}

Although the cruising speed becomes high that the windnoise is dominant, Mach number of the road vehicle is usually lower than 0.1 . Therefore, it is reasonable to use a numerical method for incompressible flow. Actually, the assumption that the pressure fluctuation is dominant for the windnoise is also based on the low-Mach-number fact.
In HFIUS, the calculation was done using second order upwind scheme and central differencing scheme.In addition, simulations were carried out in 3-dimensional, incompressible and steady conditions. The convergence level for residuals was set to $10^{-5}$ with simple pressure-velocity coupling.

\section{RESULTS AND DISCUSSION}

In this investigation, for the first step, the airflow simulations were carried out for slanted sharp-edged model and semi-small ellipsoidal model in order to understand the mechanics of airflow around vehicle Apillar. Then, the CFD results were used to estimate local sound source strength by using Boundary Layer Noise Source Model. It is essential to note that results were obtained through the lines plotted on the side window of the vehicle.

\section{SLANTED SHARP-EDGED MODEL}

Airflow pathlines analysis for slanted sharp-edged model at 0-degree yaw angle showed that the A-pillar vortex was originated from the A-pillar base. This vortex was circulating diagonally and moving downstream to the flow (Fig. 3).

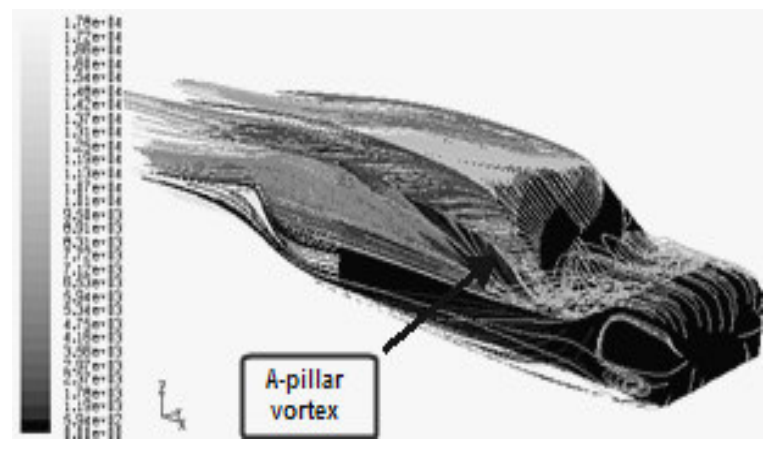

Fig. 3: Pathlines, $140 \mathrm{~km} \mathrm{~h}^{-1}$, 0' yaw angle (slanted sharp-edged model)

Results also showed that A-pillar vortex separation and reattachment region at 0 degree yaw angle slanted sharp-edged model was approximately 45 degree with respect to the A-pillar. In comparison to the past studies about mechanics of airflow for rectangular model ${ }^{[12]}$, it can be concluded that the A-pillar vortex of the slanted sharp-edged model which is circulated downstream is at a more acute angle. Therefore, a greater magnitude of 
turbulent kinetic energy existed in a smaller region. This caused the core of the A-pillar vortex became closer to the front side window. The results of relative pressure contour exhibited a decrease in negative magnitude along A-pillar base to the roof (along $\mathrm{z}$ axis). The mean pressure coefficient analysis of bottom and top row points for slanted sharp-edged model at 0 degree yaw angle showed steep decrease in $\mathrm{Cp}$ values during the first five points with minimums of -2.07 and -1.06 respectively, a strong evidence of an intense Apillar vortex (Fig. 4).

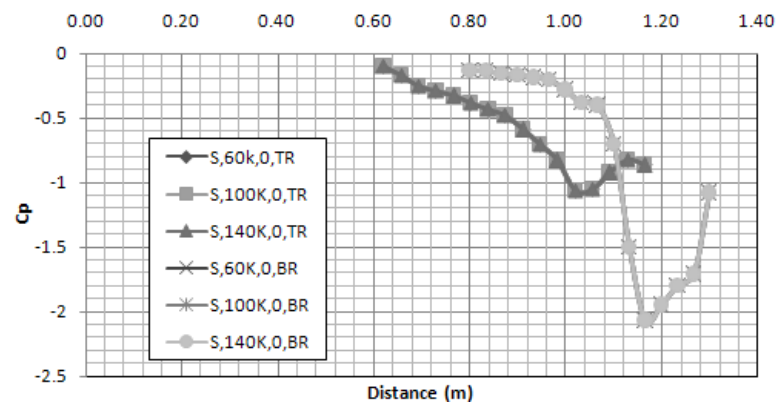

Fig. 4: Surface mean $\mathrm{Cp}$ variation with distance, $0^{\circ}$ yaw angle (slanted sharp-edged model)

Yaw angles have significant effects on the magnitude and size of the A-pillar flow separations. Different yaw angle exhibited different A-pillar vortex at varying intensity and size on each sides of the vehicle. Using the 0 yaw angle as a datum, the visualization of 15 degree yaw angle in leeward side showed that the A-pillar vortex and wake was somewhat larger than the 0 degree yaw angle condition (Fig. 5).

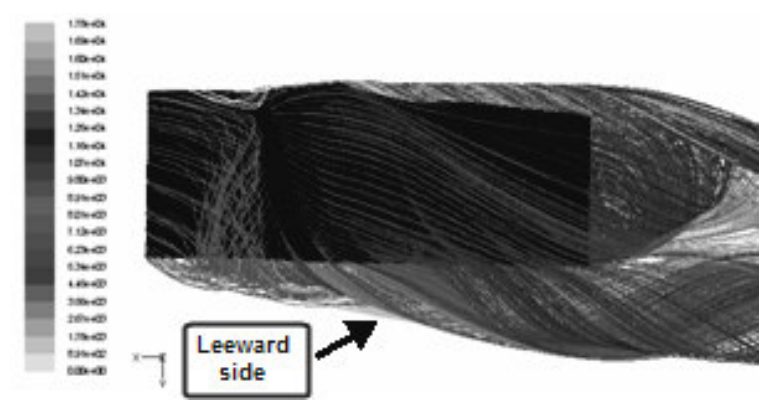

Fig. 5: Pathlines, $140 \mathrm{~km} \mathrm{~h}^{-1}, 15^{\circ}$ yaw angle, Top view (slanted sharp-edged model)

Moreover, in the windward side, the region of separated zone decreased enormously compared to leeward side. Further analysis revealed that the A-pillar vortex, which is formed at 5 and 10-degree yaw angle, could sustain its intensity only for a short distance before reattaching itself to the side window wall.

As it was evident from pathline analysis and relative pressure contour, for the slanted sharp-edged model at different yaw angles, the airflow separation started on the side window on the windward side of the flow and for the leeward side, separation starts form A-pillar to the side window.Furthermore, the pressure coefficient data analysis revealed that the vortex intensity increased on the slanted sharp-edged model on leeward side from 0 degree to 15 degree yaw angles (Fig. 6 and 7).

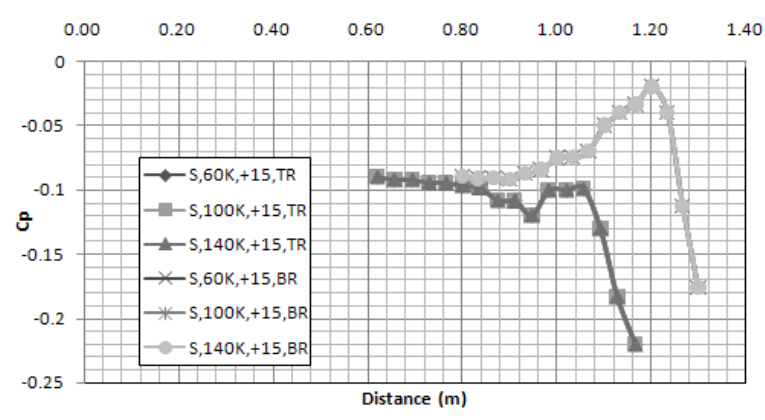

Fig. 6: Surface mean $\mathrm{Cp}$ variation with distance, $+15^{\circ}$ yaw angle (slanted sharp-edged model)

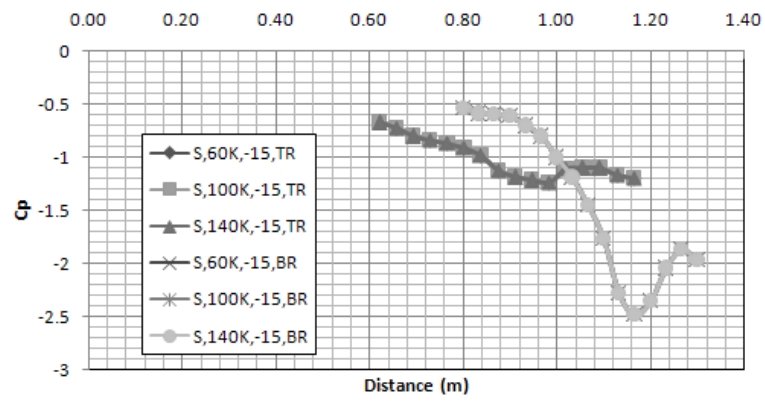

Fig. 7: Surface mean $\mathrm{Cp}$ variation with distance, $-15^{\circ}$ yaw angle (slanted sharp-edged model)

In addition, as the yaw angle increased, the location of the maximum magnitude of the pressure coefficient shifted downstream of the flow in the leeward side. Also, the plots slanted sharp-edged model showed that the surface mean pressure coefficients were relatively independent of Reynolds number at $0^{\prime}-15^{\prime}$ and +15 degrees yaw angles. 


\section{SMALL SEMI ELLIPSOIDAL MODEL}

The small semi-ellipsoidal model was made with 60 degree flat windshield inclination angle but with semi-ellipsoidal A-pillar and windshield curvature. Airflow streamline analysis in 0 degree yaw angle at different cruising speed showed that due to the small semi-ellipsoidal geometry of A-pillar/windshield, no flow separation was evident in the A-pillar region (Fig. $8)$.

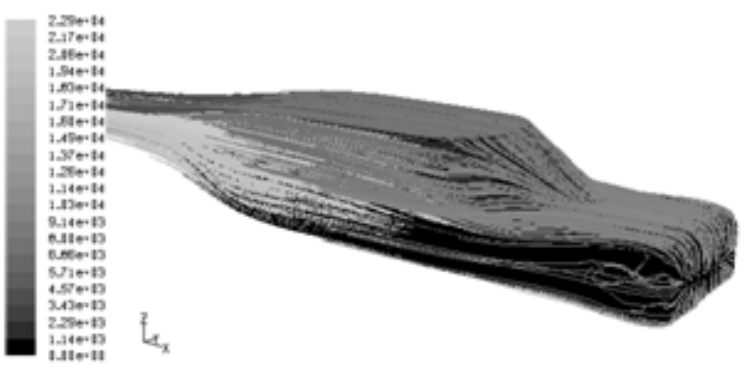

Fig. 8: Pathlines, $140 \mathrm{~km} \mathrm{~h}^{-1}, 0^{\circ}$ yaw angle (small semiellipsoidal model)

The mean pressure coefficient analysis of bottom and top row points at 0' yaw angle showed steep increase in $\mathrm{Cp}$ values during the first four points with minimums of -0.637 and -0.617 respectively (Fig. 9).

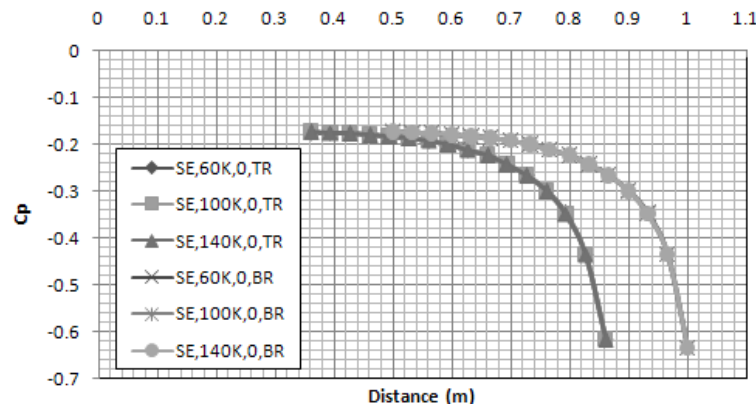

Fig. 9: Surface mean $\mathrm{Cp}$ variation with distance, $0^{\circ}$ yaw angle (slanted sharp-edged model)

In contrast, for slanted sharp-edged model, the $\mathrm{Cp}$ analysis showed steep decrease in $\mathrm{Cp}$ values during the first five points with a minimum of -2.07 , a strong evidence of an intense A-pillar votex. Yaw angles cause to form flow separation in the leeward side of this model.There were two flow separations and reattachments in this region (Fig. 10).

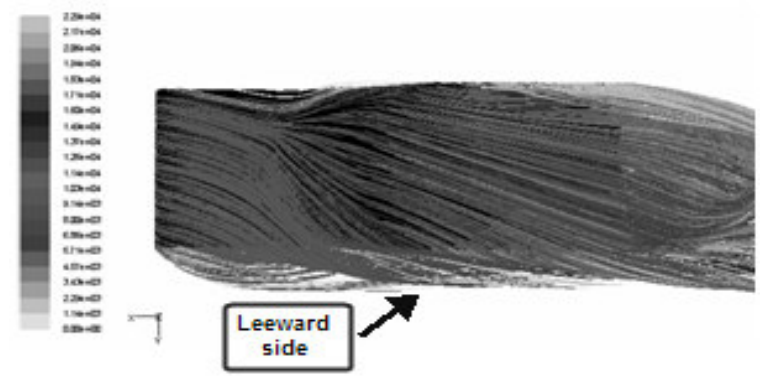

Fig. 10: Pathlines, $140 \mathrm{~km} \mathrm{~h}^{-1}$, 15' yaw angle, Top view (small semi-ellipsoidal model)

The airflow separated at the A-pillar and then reattached along a diagonal line further back on the side window. The secondary stagnation line could be considered there where the vortex flow separated on the upstream side. As it was appeared in streamline analysis, the A-pillar region flow separation for the semi-small ellipsoidal model was very small compared to the slanted sharp-edged model. The complex flow separation pattern caused to produce double peaks in mean pressure coefficient distribution in the side window. Furthermore, the magnitudes of mean pressure coefficients at the windward side were too smaller than these magnitudes at leeward side (Fig. 11 and 12).

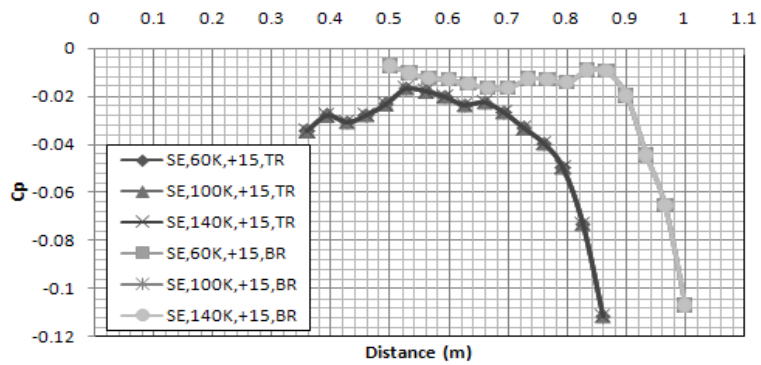

Fig. 11: Surface mean $\mathrm{Cp}$ variation with distance, $+15^{\circ}$ yaw angle (small semi-ellipsoidal model)

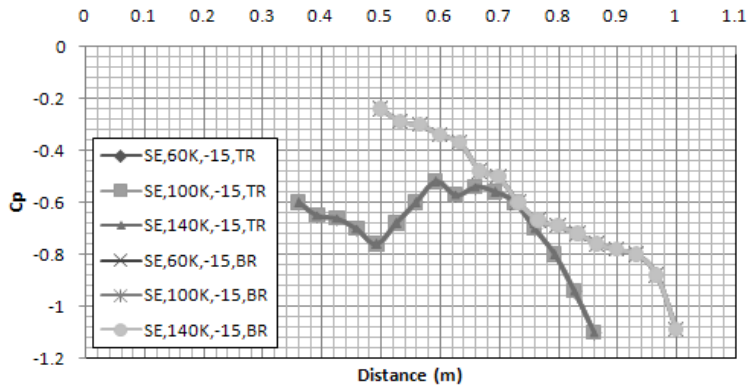

Fig. 12: Surface mean $\mathrm{Cp}$ variation with distance, $-15^{\circ}$ yaw angle (small semi-ellipsoidal model) 
The mean pressure coefficient analysis at leeward side of 15 degree yaw angle showed that the minimum value of cp was -1.08 , but the corresponding value in slanted sharp-edged model was -2.485 , a strong evidence of a more intense A-pillar vortex in slanted sharp-edged model.

\section{VALIDATION}

The results of static pressure, relative total pressure and mean surface pressure coefficient which were obtained along the A-pillar region by numerical method analysis and simulations on both simulated models were reasonable agreement with available experimental data ${ }^{[6]}$. In order to validate the method proposed in this paper, the $\mathrm{Cp}$ variation with distance for slanted sharpedged model at $140 \mathrm{~km} \mathrm{~h}^{-1}$ for 0 degree yaw angle were shown in Fig. 11 for both experimental and CFD analysis. As it was shown in Fig. 3, the CFD analysis error around A-pillar region was approximately restricted to $6 \%$, so it had a reasonable agreement with available experimental data (Fig. 13).

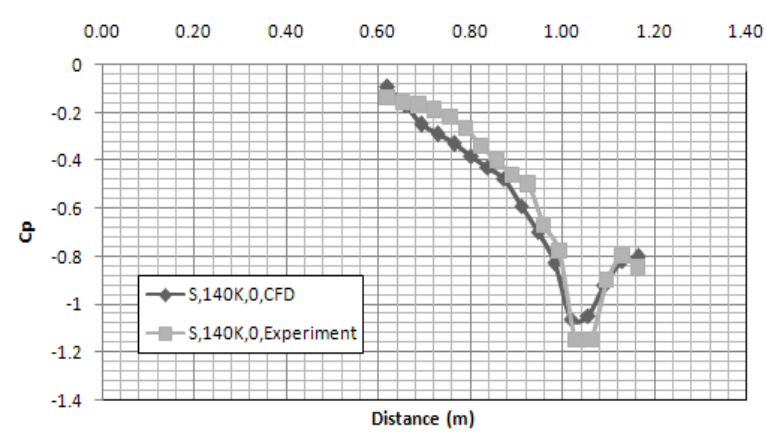

Fig. 13: Surface mean $\mathrm{Cp}$ variation with distance, 140 $\mathrm{km} \mathrm{h}^{-1}, 0^{\circ}$ yaw angle (slanted sharp-edged model)

\section{NOISE PREDICTION}

The broadband noise source model was employed in order to find the effect of A-pillar/windshield geometry to sound generated by airflow passing over the vehicle side window as a noise sources. The surface acoustic power level for slanted sharp-edged model and semi-small ellipsoidal model was shown in (Fig. 14 and 15).

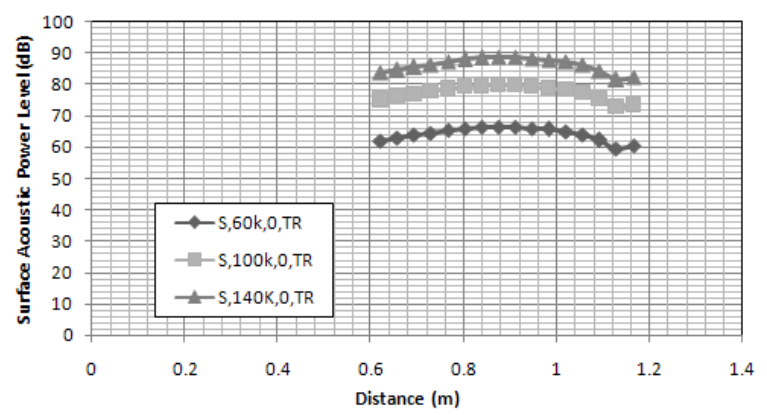

Fig. 14: Surface acoustic power level, $0^{\circ}$ yaw angle (slanted sharp-edged model)

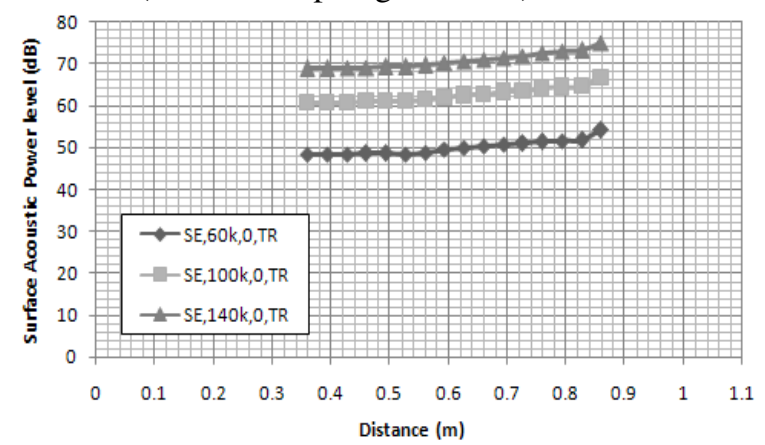

Fig. 15: Surface acoustic power level, $0^{\circ}$ yaw angle (slanted sharp-edged model)

These plots exhibited the surface acoustic power level along the top row of side window for 0 degree yaw angle at different cruising speed. Furthermore, these plots quantified the local contribution (per unit surface area) to the total acoustic power generated by the flow. It can be considered from them that the surface acoustic power level increased with increasing in cruising speed. As the windnoise is dominant at higher cruising speed, so the contours of surface acoustic power level over a side window surface of both models were shown for 0 and 15-degree yaw angle at $140 \mathrm{~km} \mathrm{~h}^{-1}$. Also, these contours quantified the local contribution (per unit-surface area) to the total acoustic power generated by the flow (Fig. 16-21).

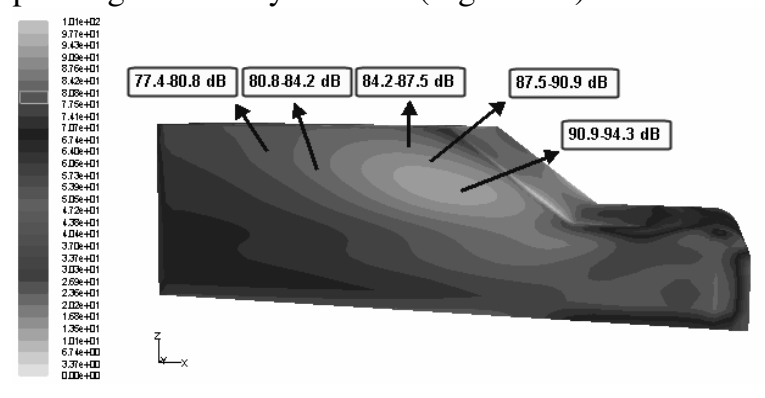

Fig. 16: Contours of Surface acoustic power level, $0^{\circ}$ yaw angle (slanted sharp-edged model) 

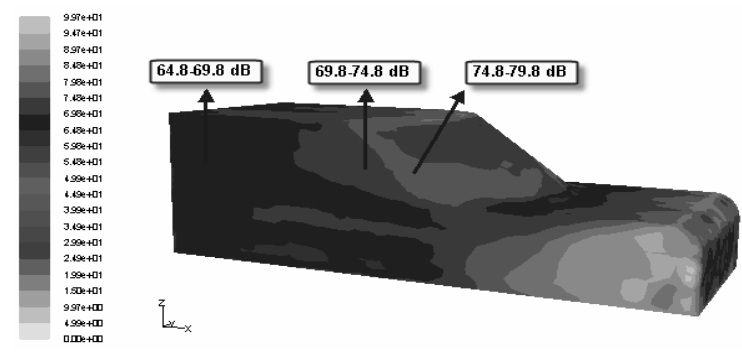

Fig. 17: Contours of Surface acoustic power level, $0^{\circ}$ yaw angle (small semi-ellipsoidal model)

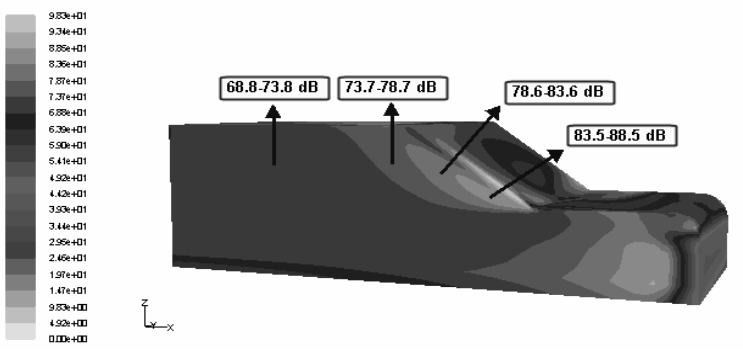

Fig. 18: Contours of Surface acoustic power level, $+15^{\circ}$ yaw angle (slanted sharp-edged model)

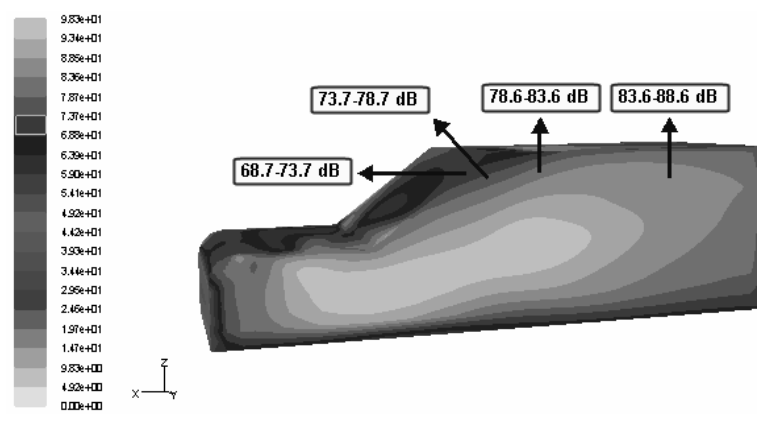

Fig. 19: Contours of Surface acoustic power level, $-15^{\circ}$ yaw angle (slanted sharp-edged model)

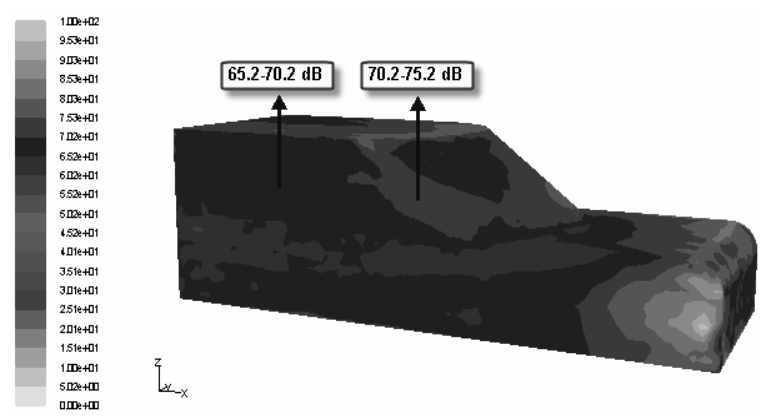

Fig. 20: Contours of Surface acoustic power level, $+15^{\circ}$ yaw angle (small semi-ellipsoidal model)

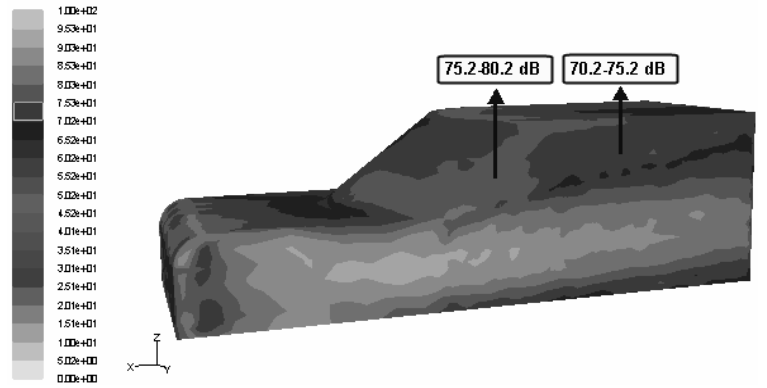

Fig. 21: Contours of Surface acoustic power level, $-15^{\circ}$ yaw angle (small semi-ellipsoidal model)

It can be considered from these counters that the maximum surface acoustic power level values for slanted sharp-edged model are larger than small semiellipsoidal model at $0^{\circ}$ and $+15^{\circ}$ yaw angle, but theses differences between maximum acoustic power level values for both models become decreased in leeward side due to complex flow sturcture of small semiellipsoidal model at negative yaw angles. Consequently, the total acoustic power emitted from side window surface of semi-small ellipsoidal model is smaller than slanted sharp-edged model.

\section{CONCLUSION}

Two simplified three-dimensional models of road vehicle were simulated by CFD in order to understand the characteristics of the airflow around A-pillar region and predict the potential of noise generation in side window surface of vehicle. In this simulation, both models were simulated at velocities 60,100 , and 140 $\mathrm{km} \mathrm{h}^{-1}$ and at 0 and 15 degrees yaw angle.

The surface mean pressure coefficients are independent of Reynolds numbers for both models for different yaw angle at different cruising speeds. At 0degree yaw angle, the airflow around the A-pillar region of slanted sharp-edged model is separated and strong vortex is produced. In contrast, the small-semi ellipsoidal model do not experience flow separation at different cruising speed for 0 degree yaw angle.

Results for slanted sharp edged model at 15 degree yaw angles show that the size of the A-pillar vortex in windward side is smaller than the leeward side. The A-pillar vortex intensity investigations in various yaw angles for slanted sharp-edged model show that vortex intensity generated in the leeward side has a higher intensity than windward side.

Results from small semi-ellipsoidal model at 15 degree yaw angle show no flow separation on windward side but minor separation is noted on leeward side. 
The vortex generated on leeward side of the slanted sharp-edged model is more intense than small semiellipsoidal model.

Broadband noise source analyses show that the A-pillar and windshield geometry and yaw angles have important effects to nature of the windnoise in vehicles. Surface acoustic power level analyses show that A-pillar and windshield local corner radii have significant effect to reduce windnoise around A-pillar region especially at different yaw angles.

\section{REFERENCES}

1. George, A.R., 1990. Automobile Aerodynamic Noise, SAE Paper, 900315.

2. Chou, S.T. and A.R. George, 1987. An analytical parametric study of the broadband noise from axial flow fans. Proceeding Noise Conference 87, Noise Control Foundation, New York, pp: 83-88.

3. Dobrzynski, W. and H. Soja, 1994. Effect on Passenger Car Wind Noise Sources of Different apost Configurations, Inter-Noise-94, Yokohama, Japan, pp: 387-390.

4. Haruna, S., T. Nouzawa, I. Kamimoto and S. Hiroshi, 1990. An Experimental Analysis and Estimation of Aerodynamic Noise Using a Production Vehicle, SAE Paper, 900316.

5. Howe, M.S., 1976. Contributions to the theory of aerodynamic sound, with application to excess jet noise and the theory of the flute. J. Fluid Mech., 71 (4): 625-673.
6. Alam, F., S. Watkins and G. Zimmer, 2002. Mean and time-varying flow measurements on the surface of a family of idealised road vehicles. J. Exp. Thermal Fluid Sci., pp: 639-654.

7. Popat, B.C., 1991. Study of Flow and Noise Generation from Car A-pillars, Ph.D. Thesis, Department of Aeronautics, Imperial College of Science, Technology and Medicine, The University of London, UK.

8. Lumley, J.L., 1978. Toward a turbulent constitutive equation. J. Fluid Mech., 41: 123-176.

9. Blazek, J., 2005. Computational Fluid Dynamic: Principles and Applications. Elsevier's London Publisher, pp: 243-247.

10. Gerolymos, G.A., 1990. Implicit multiple-grid solution of compressible navier-stokes equations using $\mathrm{k}-\varepsilon$ turbulence closure. AIAA. J., pp: 1707-1717.

11. Curle, N., 1955. The Influence of Solid Boundaries upon Aerodynamic Sound. Proc. R. Soc. London, A231: 505-514.

12. Zhu, M., Y. Hanaoka, K. Aoki and H. Miyata, 1992. Numerical prediction method of aerodynamic noise around front pillar, JSAE Parer, 921009. 